\title{
Chemical analysis and consistency characterization of domestic waste bio-asphalts
}

\begin{abstract}
The objective of this paper is to characterize the chemical and consistency characterization of the bio-binder produced from domestic waste (DWBO) as compared with conventional petroleumï asphalt binder. A petroleum asphalt was modified with DWBO at 3,6, and 9\% by weight to prepare bio-binders, respectively. Samples of the DWBO-modified binders compared to base binder were tested by running the rotational viscosity (RV). Moreover, the fourier transform infrared (FTIR) spectroscopy as well as elemental analysis tests were utilized to validate the chemical compositions and bond initiations that caused changes in stiffness and viscosity of the asphalt modified with DWBO from those of base asphalt binders. This research has revealed that there are four factors to be influenced by the use of DWBO, (i) reducing greenhouse emissions and the toxic effect of binder compared with petroleum-based asphalt binders, (ii) increasing workability, (iii) reduction in viscosity of asphalt binders which led to reduction of asphalt pavement construction costs by reducing mixing and compaction temperatures, and (iv) increased the aging induces of the control asphalt binders. Bio-oil from domestic waste was found to be a promising candidate as a modifier for petroleumï asphalt binder.
\end{abstract}

Keyword: Bio-oil; Bio-asphalt; Chemical properties; FTIR; Rotational viscosity 